\begin{tabular}{|l|l|l|l|l|}
\hline Cuadernos de Investigación Geográfica & 2000 & No 26 & pp. 95-112 & ISSN 0211-6820 \\
\hline
\end{tabular}

(C) Universidad de La Rioja

\title{
LA CUENCA EXPERIMENTAL DE RAMBLA SALADA (MURCIA) INVESTIGACIONES HIDROGEOMORFOLÓGICAS
}

\author{
F. LÓPEZ BERMÚDEZ \\ C. CONESA GARCÍA \\ F. ALONSO SARRIA \\ F. BELMONTE SERRATO
}

Área de Geografía Física. Universidad de Murcia. Campus de la Merced. 30001 Murcia

C. electrónico: lopber@um.es

RESUMEN: Clima y acción antrópica son los responsables principales de la erosión del suelo y del riesgo de desertificación que puede registrar un territorio. La estimación cuantitativa de algunos procesos hidrológicos $y$ erosivos, bajo condiciones semiáridas mediterráneas extremadas, se reali$z a$ en la cuenca experimental de Rambla Salada, situada en el centro de la Región de Murcia, sobre substrato de margas neógenas y sedimentos cuaternarios. Los usos del suelo dominantes son los de la agricultura de secano, mayoritariamente dedicada al cultivo del almendro y una cubierta vegetal, muy abierta, de especies esteparias que protegen mal al suelo. Durante el corto período de observación (1996-2000), las respuestas geomorfológicas e bidrológicas ban sido modestas debido a la sequía dominante. Bajas tasas de erosión y algunos episodios lluviosos que originaron súbitas avenidas. Un dispositivo instrumental de registro de datos a dos escalas diferentes, la utilización de un modelo digital del terreno y la integración en un entorno SIG, permite conocer y evaluar, en primera aproximación, los más destacados mecanismos geomorfológicos e bidrológicos, que operan a escalas de parcela y cuenca.

ABSTRACT: Climate and anthropogenic actions are the main.responsibles of soil erosion and desertification processes in the mediterranean semiarid regions. The quantitative estimation of some hidrological and erosives processes, under extreme semiarid climate conditions, is carry out in the experimental catcbment of Rambla Salada, located in the middle of Murcia's Region (South-East Spain), built on neogene marls and quaternary sediments. The principal land uses are associated to the dryland agricultural, mainly almond trees, and open steppe vegetation of esparto (Stippa tenaccisima) that protect badly the soil. During the short period of observation (1996-2000), the geomorpbological and bydrological responses have been poor due to drought. Low rates of soil erosion and few rainy events originated flash floods. The monitoring of catchment and a small experimental field, the use of D'TM and its integration in the GIS, allow to know and evaluate the most relevant bydrological events and geomorfological territory processes that operate to plot and catchment escales.

Palabras clave: Cuenca semiárida, Generación de escorrentía, Hidrología superficial, Cauce, Humedad del suelo, Erosión del suelo, Sedimentos, Instrumentalización.

Key words: Semiarid catchments, Runoff generation, Surface hydrology, Channel, Soil moisture, Soil erosion, Sediments, Monitoring. 


\section{Introducción}

Una de las paradojas más llamativas de las cuencas hidrológicas bajo condiciones climáticas semiáridas, es que la mayor parte de sus paisajes son resultado de la acción de los cursos de agua. Las cuencas fluviales de estos ambientes son sistemas abiertos de proceso-respuesta de un conjunto de interacciones ambientales que dan como resultado una hidrología, morfología y paisaje específicos. La magnitud, frecuencia y dinámica de estas redes hídricas suelen ser buenos indicadores de los procesos de erosión, desertificación y cambio que registran los ambientes semiáridos mediterráneos (López Bermúdez et al., 2000). Por ello, para entender la génesis y evolución del modelado de estos territorios se hace preciso comprender los procesos y las geoformas derivadas de las distintas formas de acción de la erosión por el agua. Procesos y geoformas no son partes individuales y separadas de los sistemas geomorfológicos, sino sistemas complejos interactivos en un territorio común.

La cuenca hidrográfica es, particularmente, adecuada para entender los mecanismos pluvio-hidro-geomorfológicos, por tratarse de una unidad territorial asociada a un funcionamiento hidrológico. Para entender la variabilidad espacial y temporal de las energías liberadas por las precipitaciones y las escorrentías superficiales, conocer la resistencia de litologías y suelos, y el resultado de la combinación de ambas (Graf, 1988), establecer balances hídricos, erosivos, de sedimentos, de nutrientes..., la cuenca hidrográfica se revela como el escenario más adecuado. Como el sistema fluvial de los ambientes secos opera a diversas escalas, se precisa conocer el mecanismo y la distribución espacio-temporal de las lluvias y de los flujos hídricos de la cuenca, a lo que habría que unir el impacto de las actividades humanas, notoriamente los cambios de uso del suelo.

Los modelos hidrológicos y enfoques en el estudio de generación de escorrentías en cuencas áridas y semiáridas suelen ser probabilísticos, estocásticos y determinísticos, sobre todo los dos primeros. Sin embargo, la falta de datos y la extrema variabilidad espacio-temporal de los factores que controlan las escorrentías en estas áreas, cuestionan la validez de tales modelos (Yair \& Lavee, 1985). Por ello, para explorar las relaciones entre procesos y geoformas e identificar la causalidad que controlan los factores y los cambios dichos parámetros, la instrumentalización, la teledetección, la experiencia de campo, el seguimiento y evaluación temporal son acciones y herramientas básicas en la investigación teórica y aplicada de las cuencas secas.

Se ha elegido la cuenca de Rambla Salada como ejemplo de cuenca de vaciado por procesos de erosión, de características biofísicas semiáridas y régimen hídrico efimero, torrencial y con crecidas episódicas controladas casi exclusivamente por las lluvias intensas. Es un buen ejemplo hidrogeomorfológico que registra importantes tasas de erosión, rápidas concentraciones de flujos hídricos, poderosas descargas de agua y sedimentos, recientes e importantes cambios de uso del suelo y procesos de desertificación avanzados.

\section{Situación y caracteristicas generales}

La cuenca de la Rambla Salada, entre los 595 y 60 metros de altitud y una extensión de $131,6 \mathrm{Km}^{2}$, se localiza en el centro de la Región de Murcia (Fig. 1). Se 
extiende en dirección Oeste-Este entre las localidades de Pliego y Alcantarilla con una longitud de $27 \mathrm{Km}$ y una anchura máxima de $10 \mathrm{Km}$.

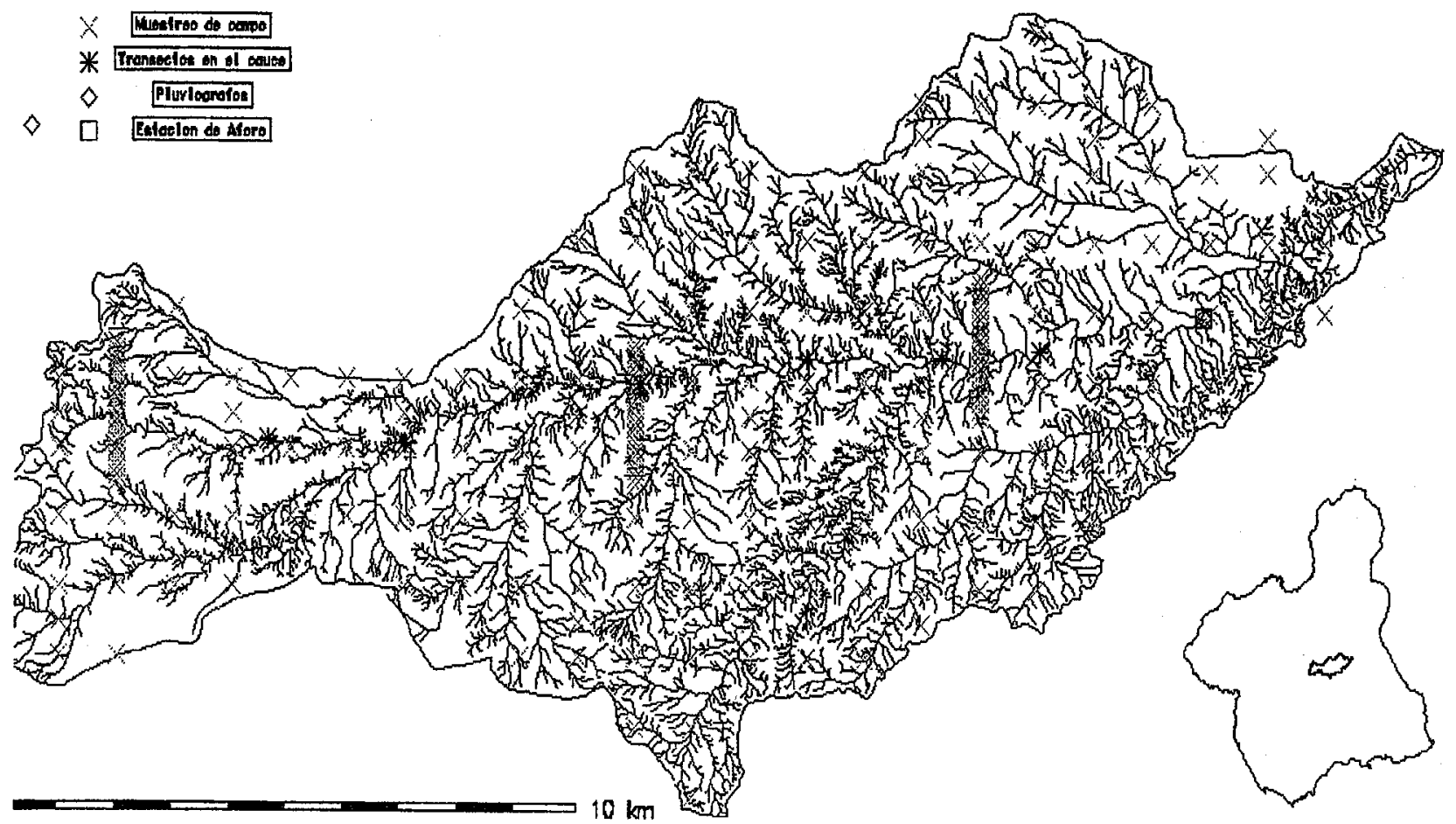

Figura 1. Cuenca de Rambla Salada.

Desde un punto de vista morfoestructural, forma parte de la cuenca sedimentaria de Mula, rellena con materiales neógeno-cuaternarios. De Oeste a Este, desde cabecera a su desembocadura aparecen en primer lugar unos relieves, asociados a formaciones carbonatadas de escasa entidad (Altos de Pliego). A continuación, dos glacis de acumulación pliocuaternarios parcialmente desmantelados por la erosión. Desmantelamiento que se acentúa hacia la confluencia con el río Segura y propicia el afloramiento de las margas neógenas, de origen marino y continental, con intercalaciones de yesos, conglomerados y areniscas.

Las geoformas dominantes resultantes de la actuación de los procesos erosivos son los badlands con barrancos en forma de V, pendientes entre 45 y 90 grados y, con frecuencia, de acusada profundidad. En el fondo de los cauces de mayor entidad aparecen formaciones cuaternarias aluviales.

Las condiciones climáticas de la cuenca se caracterizan por una temperatura media anual de $17,5^{\circ} \mathrm{C}$, temperatura media del mes más frío (Enero) de $12^{\circ} \mathrm{C}$ y temperatura media del mes más cálido (Julio) de $28^{\circ} \mathrm{C}$. La precipitación anual es muy variable, entorno a los $300 \mathrm{~mm}$ con una evapotranspiración potencial evaluada en $850 \mathrm{~mm}$. Tales condiciones someten a suelo y plantas a una fuerte tensión hídrica que se manifiesta en las bajas tasas de humedad edáfica, en el carácter esporádico de las escorrentías y en el carácter xérico de la cubierta vegetal.

La vegetación natural se compone de un matorral termófilo de algarrobo (Ceratonia siliqua,) lentisco (Pistacia lentiscus), romero (Rosmarinus officinalis), albaida (Anthyllis citisoides), tomillo (Thymus zygis), jaguarzo (Cistus albidus) y 
escobilla (Salsola genistoides). En las áreas más degradadas, afectadas por procesos de acarcavamiento más intensos, aparecen especies con mayor resilencia como Lygeum spartum, Ferula communis, Capparis spinosa y Dittrichia viscosa (López Bermúdez et al., 1992).

El cultivo tradicional más extendido, hasta mediados del siglo $\mathrm{XX}$, fue el cereal. Transformaciones posteriores disminuyeron, de modo acusado, su superficie a la vez que se producía un importante aumento de los cultivos arbóreos de secano, de almendro sobre todo. La gran expansión de este cultivo se ha producido, recientemente, al amparo de las subvenciones contempladas en la Política Agraria Comunitaria. El resultado de los cambios de uso del suelo, intensificados en los últimos años, ha sido una serie de ciclos de cultivo-abandono que han incrementado, notablemente, los procesos de erosión en gran parte de la cuenca y originado un paisaje de cárcavas, barrancos, ramblas e interfluvios en diverso grado de desmantelamiento.

\section{Recopilación y gestión de información en la cuenca.}

Gran parte de los trabajos realizados en esta cuenca se han basado en el uso de Sistemas de Información Geográfica (SIG) para el almacenamiento y gestión de la información así como para el desarrollo de modelos estadísticos y dinámicos.

Por otro lado, la cuenca de Rambla Salada se halla dotada de dos sistemas instrumentales para la evaluación y control, a distinta escala, de los episodios lluviosos e hidrológicos, la erosión del suelo y la producción de sedimentos. Un sistema automático de información hidrológica que cubre la totalidad de la cuenca y un campo experimental en ladera.

\subsection{Sistema de Información Geográfica}

El objetivo del desarrollo de un Sistema de Información Geográfica en la Rambla Salada ha sido el apoyar y dotar de cohesión a los diversos trabajos llevado a cabo en ella. Por otro lado se han generado nuevas herramientas que permiten la aplicación de nuevas técnicas de análisis espacial y modelización (Alonso Sarría, 1995).

En un principio se utilizó IDRISI en un entorno MS-DOS apoyado con el desarrollo de módulos en QuickBasic. Dadas las limitaciones de esta solución, se optó en el año 1997 por el cambio al uso de GRASS en un entorno linux y para el futuro se apuesta su combinación con PCRaster para el desarrollo de modelos dinámicos. El uso de herramientas de software libre y código abierto se justifica ampliamente por la necesidad de contar con herramientas lo más flexibles posible para el desarrollo de modelos.

El SIG se ha organizado en diversas escalas de trabajo. En primer lugar, una escala global de cuenca cuyas fuentes de información están a escala 1:50000 y 1:25000. Entre la información disponible a esta escala se incluye un Modelo Digital de Terreno de la cuenca (modelo de elevaciones y derivados), litología, suelos, imágenes de satélite y mapas de usos del suelo en diferentes años. A esta escala se ha realizado 
también un exhaustivo muestreo de campo cuyos resultados están aún siendo analizados (Fig. 1).

En segundo lugar, una escala detallada (Fig. 2) a partir de información a escala 1:5000, trabajo de campo y fotografía aérea digital (Fig. 3). El objetivo fundamental del trabajo a esta escala es el cauce principal de la cuenca. Finalmente, se ha llevado a cabo una cartografia de detalle en el campo experimental Minglanillo (Fig. 4).

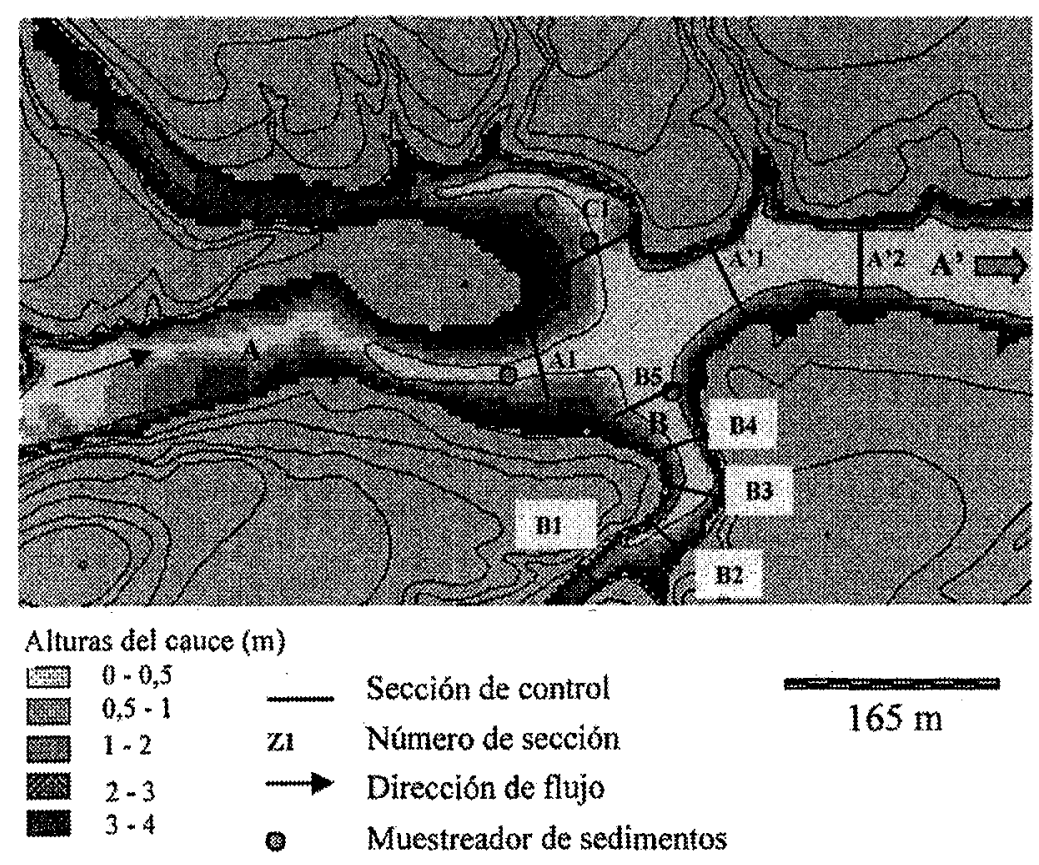

Figura 2. Localización de las secciones estudiadas en el cauce principal de Rambla Salada (A) y cauces tributarios del Barranco de la Zarza (B) y Cañada Ramblixo (C). Las alturas están referidas a los niveles de base relativos del talweg principal.

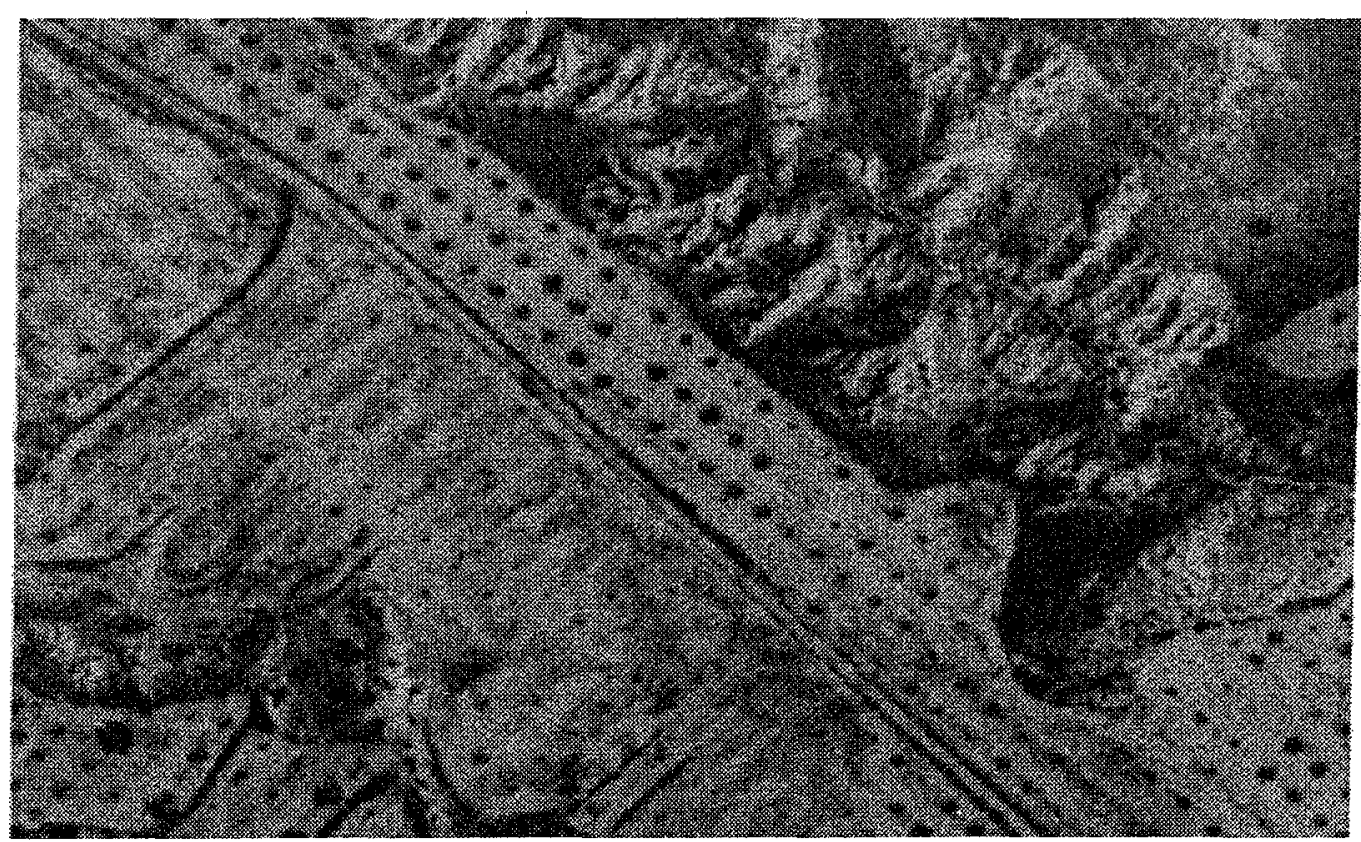

Figura 3. Fotografía aérea digital de detalle en Rambla Salada en donde puede apreciarse el cultivo de almendros en terrazas y ocupando el fondo de las vaguadas, y la fuerte erosión de las laderas. 


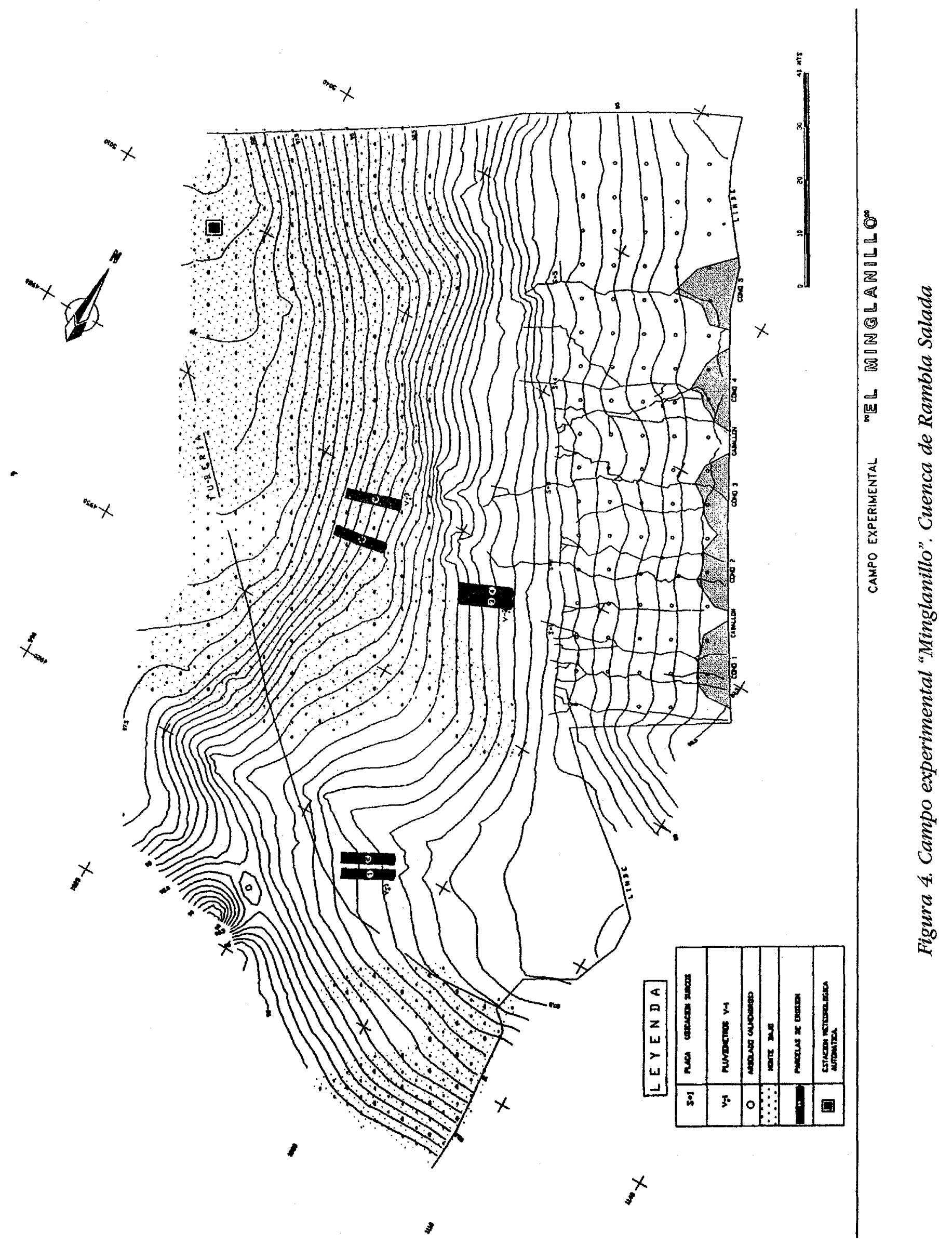




\subsection{El Sistema Automático de Información Hidrológica (SAIH)}

Es una red telemétrica de captación de datos hidrológicos e hidraúlicos, un sistema integrado de información mediante el cual, en tiempo real, se puede conocer en un punto o puntos determinados de una cuenca hidrográfica las variaciones de las características hidrológicas e hidraúlicas, a la vez que permite disponer de estadísticas hídricas más completas y fiables y agilizar la toma de decisiones respecto a dos tipos de problemas principales (Cabezas, 1989; C.H.S., 1992; Pedrero González, 1993): (a) Previsión y seguimiento de la evolución de las avenidas, para prevenir y minimizar daños; (b) Gestión de los recursos hidrológicos a fin de optimizar su asignación y explotación, especialmente en las situaciones de escasez a corto y medio plazo (sequías).

El principal objetivo de la red pluviométrica del sistema SAIH, es proporcionar datos con la suficiente resolución espacial y temporal como para predecir los caudales de avenida a través de modelos precipitación-escorrentía (Cabezas, 1988; García,2000). El sistema, pues, puede ser considerado como un potente instrumento de trabajo para los fenómenos de gran variabilidad espacio-temporal como son las precipitaciones y flujos hídricos de los ámbitos semiáridos, donde ríos-rambla y ramblas son los cursos fluviales dominantes. Los datos registrados también pueden servir para evaluar tasas de erosión del suelo y producción de sedimentos.

El sistema permite una recepción silmultánea, y en tiempo real, de la información capturada por sensores remotos ubicados en toda la cuenca. El sistema tiene una estructura jerárquica de tipo arborescente, apoyada en los siguientes tres niveles:

- Puntos de control para captación de datos. Se trata de una red de más de 300 sensores distribuidos en 92 puntos de control para la medida de las variables físicas: precipitaciones acumuladas e intensidades con frecuencia programable entre un máximo de 1 día y 5 ó 10 minutos, evaporación directa o calculada, temperaturas, viento, radiación, etc; control de la altura de la lámina de agua, velocidad y caudales (caudalímetros ultrasónicos, piezorresistivos y potenciómetro) y, en algunos casos, calidad de las aguas en ríos, canales y ramblas; puntos de control en presas para captar las variables de gestión de embalses; puntos de control telenivómetro para medir alturas y densidades de nieve acumulada; puntos de control del nivel freático para medir la altura y calidad del agua subálvea. La cuenca de Rambla Salada dispone de un punto de control próximo a la confluencia con el río Segura. Otros cuatro puntos de control se ubican en las contiguas cuencas del río Mula (al Norte) y rambla de Algeciras (al Sur).

- Puntos de concentración. La red de puntos de control se halla dividida en distintas áreas, en cada una de las cuales se ha seleccionado uno, conocido como punto de concentración cuyas funciones son las de recibir la información desde los puntos de control, llevar a cabo un primer tratamiento informático de los datos y transmitirlos al Centro de Proceso de la Cuenca. Estos puntos de proceso local, siete en la Cuenca del Segura, suelen estar situados en los embalses principales o en las oficinas regionales de la Confederación Hidrográfica.

- Centro de procesamiento de datos de la cuenca. Es el nivel superior del sistema, órgano de control del que emanan las recomendaciones para la decisión. Consti- 
tuye, a la vez, un centro de estadística hidrológico/hidraúlica que recibe y archiva, automáticamente, la información desde todos los puntos de concentración. Procesa los datos mediante programas y modelos. Calibra los modelos de simulación y trabaja en el perfeccionamiento progresivo de sistemas expertos para el apoyo en la toma de decisiones. Aplica las decisiones de explotación tomadas por los responsables de la cuenca (Pedrero González, 1993).

\subsection{El campo experimental de "Minglanillo"}

Este campo experimental se halla incluido en la Red de Estaciones Experimentales de Seguimiento y Evaluación de la Erosión del Proyecto LUCDEME (RESEL) de la Dirección General de Conservación de la Naturaleza, Ministerio de Medio Ambiente (Rojo y Sánchez Fuster, 1996). Se localiza en los $37^{\circ} 57^{\prime} 30^{\prime \prime} \mathrm{N}$ y $1^{\circ}$ $25^{\prime} 20^{\prime \prime}$ Oeste, en una ladera a 350 metros de altitud, orientada al Norte $45^{\circ}$ y pendiente entre el 24 y el 5 por ciento. El substrato rocoso está constituido por margas neógenas que soportan un suelo regosol margálico recubierto, parcialmente, por un matorral xérico, con el esparto (stipa tenacissima) como planta dominante en la parte superior y por cultivo de almendros en la inferior (Fig. 4). Las condiciones climáticas de esta área son algo más extremadas que las del conjunto de la cuenca. La precipitación media anual oscila entre los 250 y $300 \mathrm{~mm}$, la temperatura media es de $17,5^{\circ} \mathrm{C}$ y la evapotranspiración potencial anual, es de $900 \mathrm{~mm}$.

Uno de los recursos más extendidos para obtener información hidrológica y geomorfológica de cierto valor comparativo, lo constituyen las parcelas experimentales. Su seguimiento y control permiten comparar el efecto de diferentes factores topográficos (pendiente, exposición, desarrollo de la ladera), de las características físico-químicas del suelo, de la pedregosidad o de la estructura y densidad de la cubierta vegetal sobre la pérdida de suelo y el funcionamiento de la escorrentía. Identificar umbrales en el volumen e intensidad de precipitaciones para producir escorrentías y sedimentos, analizar procesos hidrológicos tales como las tasas de infiltración o las características de generación de escorrentía y comparar los efectos erosivos de diferentes usos del suelo y técnicas de laboreo (López Bermúdez et. al.,1993).

Para la consecución de estos objetivos, el campo se halla dotado con una estación meteorológica automática dotada de pluviógrafo, termómetro, higrómetro, radiómetro y veleta-anemómetro. Tres pares de parcelas de erosión de 2 x 10 metros con distinto uso del suelo y un transecto de control de humedad del suelo mediante TDR. Además, se controla la evolución de la red de surcos de una parcela de almendros de $34 \times 100 \mathrm{~m}$, mediante perfiladores microtopográficos.

Los coeficientes de escorrentía y la producción de sedimentos registran una alta variabilidad en estrecha correlación con las características de las lluvias y con la humedad antecedente del suelo. Así, por ejemplo, en el año 2000, los $270 \mathrm{~mm}$ de lluvia registrados fueron originados por 17 eventos, de los cuales sólo 9 produjeron lluvia con volumen e intensidad suficiente para generar escorrentía y movilizar sedimentos. Tan sólo dos episodios, acaecidos el 29 de Septiembre y el 24 de Octubre, con precipitaciones de 38 y $65,8 \mathrm{~mm}$, respectivamente, generaron entre el 75 
y el $80 \%$ de los sedimentos anuales y entre el 50 y el $70 \%$ de la escorrentía. Resultados que confirman mecanismos conocidos para estos ambientes áridos, la concentración de los episodios erosivos en unos pocos sucesos de lluvias o en otras palabras, los aguaceros extremos son los responsables de la mayor parte de la remoción y exportación de sedimentos.

Durante el período de observación (1996-2000) las tasas de erosión registradas han sido moderadas. Es la respuesta a la escasez y baja energía de las lluvias registradas y, en menor cuantía, a los usos del suelo. En las parcelas cultivadas los valores alcanzados oscilan entre las 6 y $15 \mathrm{t} / \mathrm{ha} / \mathrm{año}$ En las parcelas con matorral las tasas registradas son muy bajas, entre 0,7 y $1,4 \mathrm{t} / \mathrm{ha} / \mathrm{año}$, mientras que aquellas ubicadas en campo de cultivo abandonado las pérdidas de suelo oscilan entre 0,14 y 2 t/ha/año. Valores que guardan una buena correlación con la escorrentía generada, notoriamente, durante los aguaceros de alta intensidad (Fig. 5). Sin embargo, las bajas tasas de pérdida de suelo obtenidas parecen contradecir la constatación de que en los ambientes semiáridos la erosión, transporte y sedimentación sean el proceso geomorfológico más activo. Por ello, en la valoración de estas débiles valores conviene tener en cuenta la sequía dominante en el período analizado y el efecto de la escala sobre los procesos de erosión. A distintas escalas de observación, diferentes tasas de erosión. Los valores medios globales para la totalidad de la cuenca, en fase de evaluación, arrojaran tasas bastante superiores a las citadas.

La formación de surcos en la pärcela de almendros, iniciado en 1996, conocidas las características físico-químicas del suelo, se realiza mediante el control de sus geometrías, trazados, morfometría. Estos atributos geométricos son analizados con un SIG, prácticas agrícolas (frecuencia, dirección y profundidad de labrado) y variables

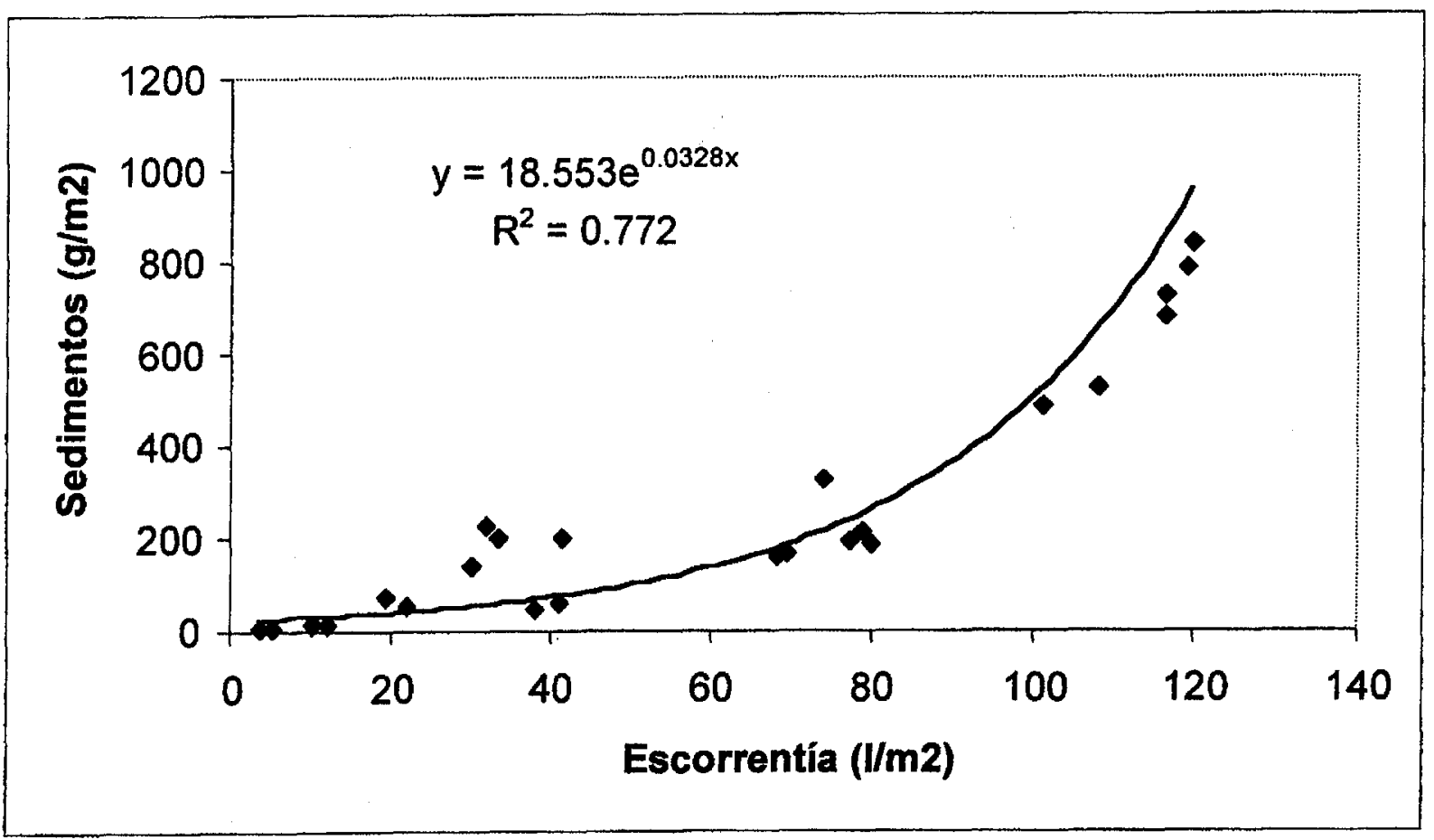

Figura 5. La producción de sedimentos expresa una correlación exponencial con la escorrentía, mostrando la importancia que los acontecimientos lluviosos extremos tienen en la erosión de los suelos en estos ambientes semiáridos extremados. 
climáticas. Cada evento de lluvias genera una escorrentía superficial rápida, normalmente asociada a flujos hídricos subsuperficiales y fenómenos de micropiping. La evolución de la red se sigue tras cada suceso pluviométrico relacionándola con las condiciones hidraúlicas de la corriente y con las tasas de concentración de humedad del suelo.

Con el tiempo podrán establecerse umbrales de intensidad pluviométrica relacionados con la generación y desarrollo de surcos, estimar pérdidas de suelo y definir el grado de afección de determinadas prácticas agrícolas en la génesis y evolución de estas geoformas indicadoras de la degradación del suelo.

\section{Distribución espacio-temporal de las lluvias.}

Uno de los elementos fundamentales en la dinámica hidrológica y geomorfológica de los sistemas fluviales en condiciones semiáridas es la enorme variabilidad espacio-temporal de los episodios de precipitación.

Se ha realizado la modelización espacio-temporal de los mismos con resultados bastante poco concluyentes. Los intentos de aplicar técnicas geoestadísticas no han dado buenos resultados debido tanto a la inadecuada densidad de datos (pluviómetros) como a la dificultad de ajustar modelos sencillos a unos datos tan complejos (Alonso Sarría, 1992; Alonso Sarría y López Bermúdez, 1994).

Respecto a la estructura de variabilidad temporal se ha puesto en evidencia un comportamiento fractal de los episodios de precipitación (Alonso-Sarría, 1995) a escala temporal media (horas-meses) y un comportamiento aleatorio a escalas más reducidas.

\section{Análisis topográfico y geomorfológico}

Se cuenta con un análisis topográfico y geomorfológico mediante técnicas de modelización cartográfica en un entorno SIG. El primer paso fue la generación de un Modelo Digital de Elevaciones a partir de las curvas de nivel del mapa 1:25000. A partir de este MDE se generaron diferentes capas de información topográfica, morfométrica e hidrológica (pendiente, curvatura en planta, orientación, área drenada, etc.) Respecto a la capa de pendientes se utilizaron dos métodos, el primero calcula la pendiente máxima, el segundo calcula la pendiente en la dirección del flujo. Se obtiene también la distancia al pixel al que desagua (Alonso Sarría et al., 1998)

\section{Evolución de los usos del suelo}

Los usos del suelo tienen una importante influencia en la generación de escorrentías y en las tasa de erosión. En la cuenca de Rambla Salada, a pesar de la falta de información oficial sobre los usos del suelo, puede inferirse, a partir de datos de archivo y campo, una primera dedicación al secano cerealista con algo de 
regadío tradicional. En este esquema, los relieves orientales aparecerían cubiertos de bosque mixto, las zonas mejor conservadas de los glacis por cultivo de cereal mientras que cárcavas y barrancos acogerían formaciones de matorral semiárido. Cabe destacar la presencia en los Altos de Pliego de un pequeño acuífero que aportaba un caudal modesto pero relativamente constante a la rambla. En el cauce principal se pueden ver todavía los restos de un pequeño molino que -elevaría el agua y permitiría el regadío de huertas de escasa extensión, el bombeo de las aguas subterráneas acabó con estos flujos superficiales.

El análisis de esta evolución (Romero Díaz et al., 1998) se ha llevado a cabo a partir de información recogida para los años 1956 (fotografía aérea escala 1:30000), 1978 (mapa topográfico escala 1:25000) y 1997 (reconocimiento detallado de campo). Los resultados muestran unas diferencias muy marcadas, notoriamente si se comparan los resultados de 1956 con los obtenidos cuarenta años después. Se registra una reducción de la superficie dedicada a matorral y sobre todo al cereal, acompañada de un incremento del arbolado de secano (almendro y en menor cuantía olivar) que pasa de un 11,5\% de la superficie en 1956 a un $50 \%$ en 1997.

La comparación de estos resultados con los mapas fisiográficos de la cuenca, permite obtener diversas conclusiones respecto a la relación que aparece entre topografía y evolución de los usos del suelo. En primer lugar, hay un descenso paulatino en altitud de pinares y matorrales, esto indica un proceso de abandono del suelo cultivado en las áreas menos favorecidas. Por otra parte, la superficie destinada al cereal aumenta su altitud media, lo que indica una progresiva marginalización de este uso. La combinación entre un mal uso y gestión del suelo y su abandono posterior suele ocasionar, en ambientes secos con lluvias muy irregulares y frecuentemente violentas, un incremento de los procesos de erosión y degradación del suelo.

\section{Régimen de avenidas}

Como las demás ramblas que surcan los terrenos semiáridos del Sureste peninsular, la Rambla Salada funciona de forma intermitente a través de sucesos hidrológicos discretos en el tiempo. Largos períodos de cauce seco son interrumpidos por avenidas, en ocasiones extraordinarias, que reactivan la morfología del lecho, readaptando la carga de fondo y la pendiente longitudinal a las nuevas condiciones ambientales.

Estas avenidas están asociadas a lluvias de alta intensidad, en unos casos de origen convectivo (avenida del 18-19 de junio 1997), en otros provocadas por la presencia de "gotas frías" que mantienen una gran inestabilidad en altura y refuerzan, por ascendencia dinámica, las elevaciones frontales de las capas bajas (avenidas del 2930 de septiembre 1999 y 23 de octubre 2000). Con menos frecuencia aparecen borrascas ligadas a sistemas frontales del Oeste, como la ocurrida en febrero de 1999.

La mayoría de los hidrogramas de avenidas registradas, en el tramo bajo de Rambla Salada (estación del S.A.I.H.), pertenecen al tipo 'flash flood'. Muestran la forma de un único pico prácticamente vertical en la fase ascendente para caer también rápidamente en el transcurso de unas horas o a lo sumo de un día. Los ejemplos más 
claros los constituyen los hidrogramas del 29-30 de septiembre 1997 y del 7-8 de septiembre 1999. En apenas media hora se alcanza el caudal pico $\left(87,8 \mathrm{~m}^{3} / \mathrm{s}\right.$ y 48,3 $\mathrm{m}^{3} / \mathrm{s}$ respectivamente) para descender de forma rápida en menos de 11 horas. La misma forma adopta el hidrograma del 18-19 de junio 1997, aunque al pico principal $\left(14,9 \mathrm{~m}^{3} / \mathrm{s}\right)$ le precede otro aislado más pequeño de una hora de duración. Los excepcionales volúmenes precipitados en intervalos tan cortos de tiempo, originan un considerable y repentino aumento de la escorrentía superficial debido al estado extremadamente seco del suelo y su escasa capacidad de infiltración durante fuertes aguaceros. Estas condiciones generan flujos hortonianos que son particularmente significativos cuando la alta intensidad de la lluvia perdura varias horas.

Sólo la avenida del 27-28 de febrero 1999 (Fig. 6), presenta un tipo de hidrograma diferente, con dos picos de caudal, uno principal $\left(123 \mathrm{~m}^{3} / \mathrm{s}\right)$ y otro secundario $\left(18 \mathrm{~m}^{3} / \mathrm{s}\right)$. Además muestra una rama ascendente algo más suave, pasando de 40 a $65 \mathrm{~m}^{3} / \mathrm{s}$ en tres horas. El intervalo entre ambos picos es de 8 horas y la duración total de la escorrentía rebasa las 20 horas.

Se ha utilizado el Sistema de Información Geográfica de cuenca para estimar los parámetros del modelo de Hidrograma Unitario Geomorfológico. Los resultados muestran que para el total de al cuenca, $131.6 \mathrm{Km}^{2}$, el parámetro es de 3.155 y el parámetro K 109.4. El hidrograma resultante tiene un tiempo de retardo algo mayor que el que aparece en los hidrogramas generados a partir de datos del SAIH, sin embargo hay que tener en cuenta el retardo que sobre las escorrentías puede suponer la infiltración en cuencas semiáridas debido tanto a la escasa humedad del suelo como a episodios de precipitación caracterizados por súbitos máximos de precipitación.

\section{Dinámica geomorfológica de los cauces}

Las ramblas ajustan la geometría de su cauce y perfil longitudinal, principalmente, de acuerdo con la frecuencia y magnitud de los sucesos hidrológicos. De acuerdo con la clasificación hidromorfológica empleada por Harvey et al. (1982) y Conesa García (1995) para este tipo de sucesos existen: (a) menores, con ligeros cambios locales en el lecho;(b) moderados, que alteran la morfología del cauce principal, y (c) mayores, que afectan a todo el sistema fluvial. Al primer grupo, pertenecen, dentro del período estudiado (noviembre 1996 - diciembre 2000) un total de 9 sucesos, 6 al segundo y 4 al tercero.

La cuenca de Rambla Salada ha experimentado en las últimas décadas una importante alteración medioambiental : cambios de los usos del suelo y constricciones del cauce debidas al canal de regadío del postrasvase Trasvase Tajo Segura que han modificado sensiblemente el régimen de la corriente y su grado de efectividad geomorfológica, causando nuevos ajustes en los cauces. Las perturbaciones que sufren los tributarios de la rambla han terminado por alterar el estado de equilibrio del cauce principal. Aguas abajo de la confluencia de estos cursos laterales, el cauce de Rambla Salada se ve forzado a adoptar el nivel de base local impuesto por los cursos que han tenido una respuesta más agresiva a dichos cambios. De sus dos tributarios principales, Barranco de la Zarza por la margen derecha y Cañada Ramblizo por la parte izquierda, el primero muestra un mayor gradiente y un lecho menos 


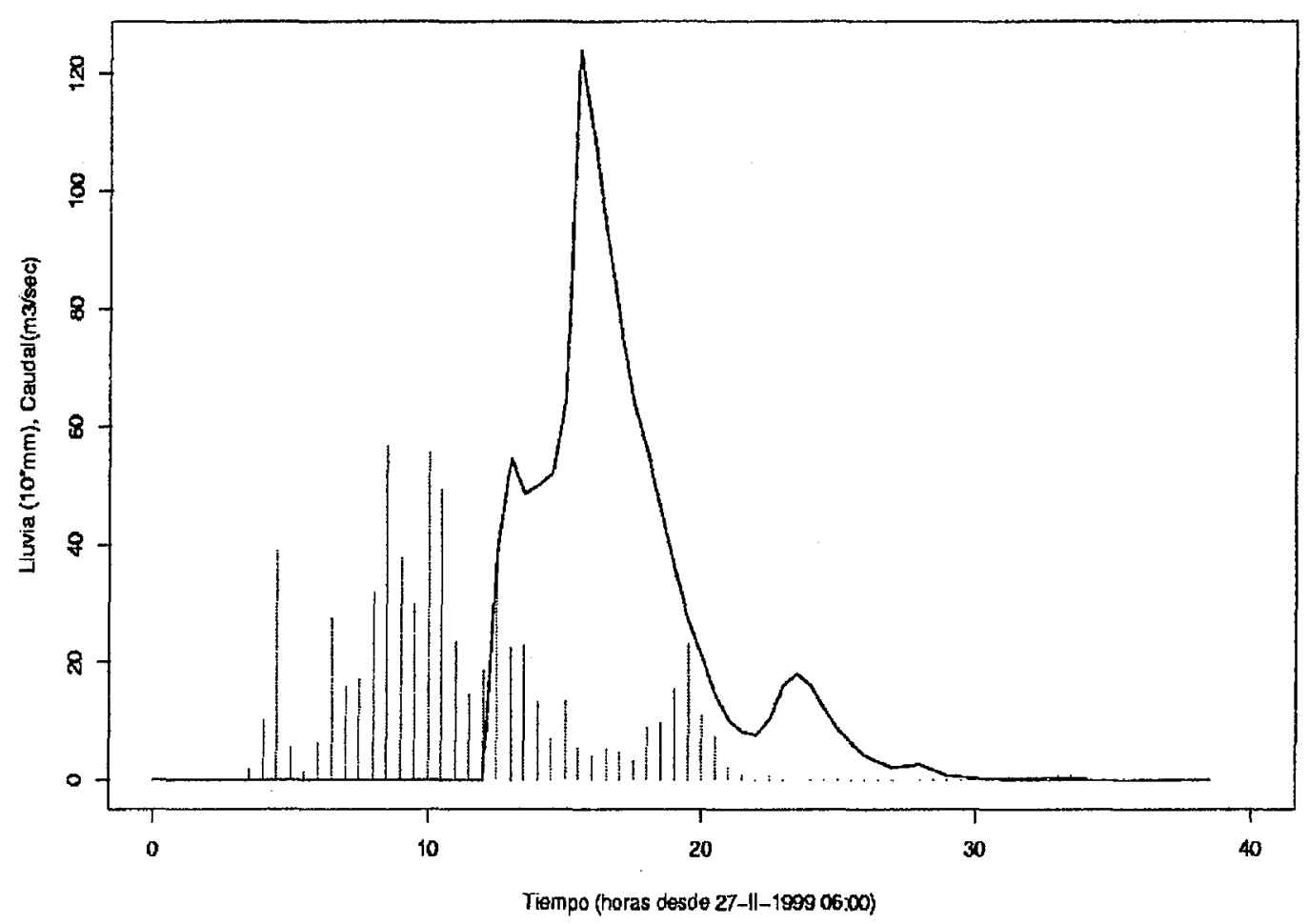

Figura 6. Histograma e bidrograma del episodio lluvioso y avenida de Febrero de 1999

uniforme, constituido por material heterogéneo grueso, que sugiere mayores tasas de energía y transporte de fondo. Su nivel de base actual controla el de Rambla Salada, que tiende a ser rebajado a partir de una erosión regresiva aguas arriba. Cañada Ramblizo, en cambio, dibuja un arco de amplio radio de curvatura antes de unirse a Rambla Salada, con escasa pendiente y mayor cobertura vegetal en su área de drenaje. Ofrece en conjunto un carácter geomorfológico más estable.

8.1. Efectos de los aportes laterales de agua y sedimentos sobre el cauce principal de Rambla Salada.

Se han relacionado los procesos de erosión del cauce y el movimiento del material de lecho bajo diferentes regímenes de corriente, aguas arriba y abajo de la confluencia del Barranco de la Zarza con Rambla Salada. Para ello se han elegido cuatro tramos en torno a este punto: tramo A (Rambla Salada aguas arriba de su unión con Cañada Ramblizo y Barranco de la Zarza), A' (Rambla Salada aguas abajo de este punto), B (Barranco de la Zarza aguas arriba de su confluencia con el cauce principal) y C (Cañada Ramblizo, inmediatamente aguas arriba del mismo punto) (Fig. 2).

a) Movilidad del material de lecho. Los modelos de cauce de los tramos experimentales provistos de lechos de gravas, tramo B, curvilíneo, en el Barranco de la Zarza, y tramo A', rectilíneo, en Rambla Salada, han obligado a establecer diferente número de secciones para cada tramo. En la curva $\mathrm{B}$ se eligieron cinco secciones, mientras que el tramo A', al ser muy poco sinuoso, sólo ha requerido dos secciones. Para controlar el movimiento de las partículas en el lecho se han colocado juegos de trazadores identificados por colores que representan las distintas secciones y números referentes a su posición dentro de éstas. 
A partir de los trabajos de campo realizados, hasta ahora, se han constatado varios hechos de especial interés.

- La influencia de las posiciones de partida sobre las longitudes y probabilidades de transporte. Las primeras medidas parecen indicar que las pozas son lugares preferenciales de deposición, pero tienen escasa importancia como áreas fuente de formas de lecho. En aguas bajas, los cantos situados en estos lugares muestran las menores longitudes de transporte, pero en condiciones de régimen alto las pozas favorecen, o al menos no impiden, la remoción de la carga de fondo, como ya han puesto de manifiesto Schmid \& Gintz (1995).

- La influencia de la forma de las partículas. Las distancias de desplazamiento y sus posiciones son correlacionadas con las clases de formas de Zingg: 1. discoidal, 2. esférica, 3. elipsoide y 4. cilíndrica. En general, esta influencia se deja sentir en sucesos moderados y de baja magnitud, siendo las formas esféricas las que experimentan mayor desplazamiento ante una misma tensión media de corte, seguida de las cilíndricas, elipsoides y discoidales. Para las avenidas extremas no se ha constatado una especial relación entre la distancia de recorrido y la forma de las partículas.

b) Erosión y sedimentación del cauce. Para cuantificar el balance de erosiónsedimentación en cada sección transversal se instalaron clavos de erosión en diferentes formas de lecho (dunas, antidunas, pozas, barras laterales y longitudinales,...). El mismo procedimiento se empleó para obtener las tasas de erosión de los márgenes, colocando clavos de erosión en las porciones de margen superior e inferior. Durante el periodo de observación (noviembre 1996 diciembre 2000) se han producido importantes variaciones del lecho y de los márgenes, que están siendo objeto de estudio en la actualidad.

Entre los efectos más relevantes destacan los siguientes: 1) Erosión regresiva

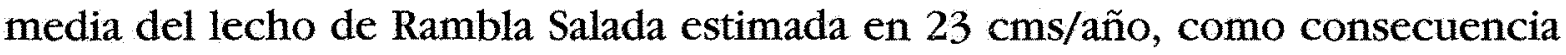
de su adaptación al nivel de base local impuesto por la afluencia del Barranco de la Zarza (Fig. 7); 2) erosión apreciable del margen derecho del Barranco de la Zarza en sus secciones próximas a la confluencia (B4 y B5); 3) extraordinaria erosión transitoria desarrollada durante las avenidas de 1997 y febrero de 1999, que supuso la destrucción de las barras centrales aguas abajo del punto de confluencia, la creación de profundas pozas y la exhumación de grandes bloques; 4) erosión progresiva del lecho principal y acreción del lecho activo de inundación aguas abajo de la citada confluencia, mereciendo reseñar el papel de la vegetación como factor de fricción a la corriente y regulador de la sedimentación.

\subsection{Cambios morfológicos y sedimentarios de los cauces}

La identificación de discontinuidades en las formas de lecho y en la variación de tamaños de las partículas a lo largo de Rambla Salada incluye la consideración de los efectos del suministro de sedimentos por parte de los tributarios, que hacen variar las tasas de erosión y transporte en función de la litología que atraviesan y los impactos realizados por el hombre. La naturaleza litológica de las laderas drenadas y las distancias recorridas a partir de las áreas fuente de sedimentos son dos factores muy 


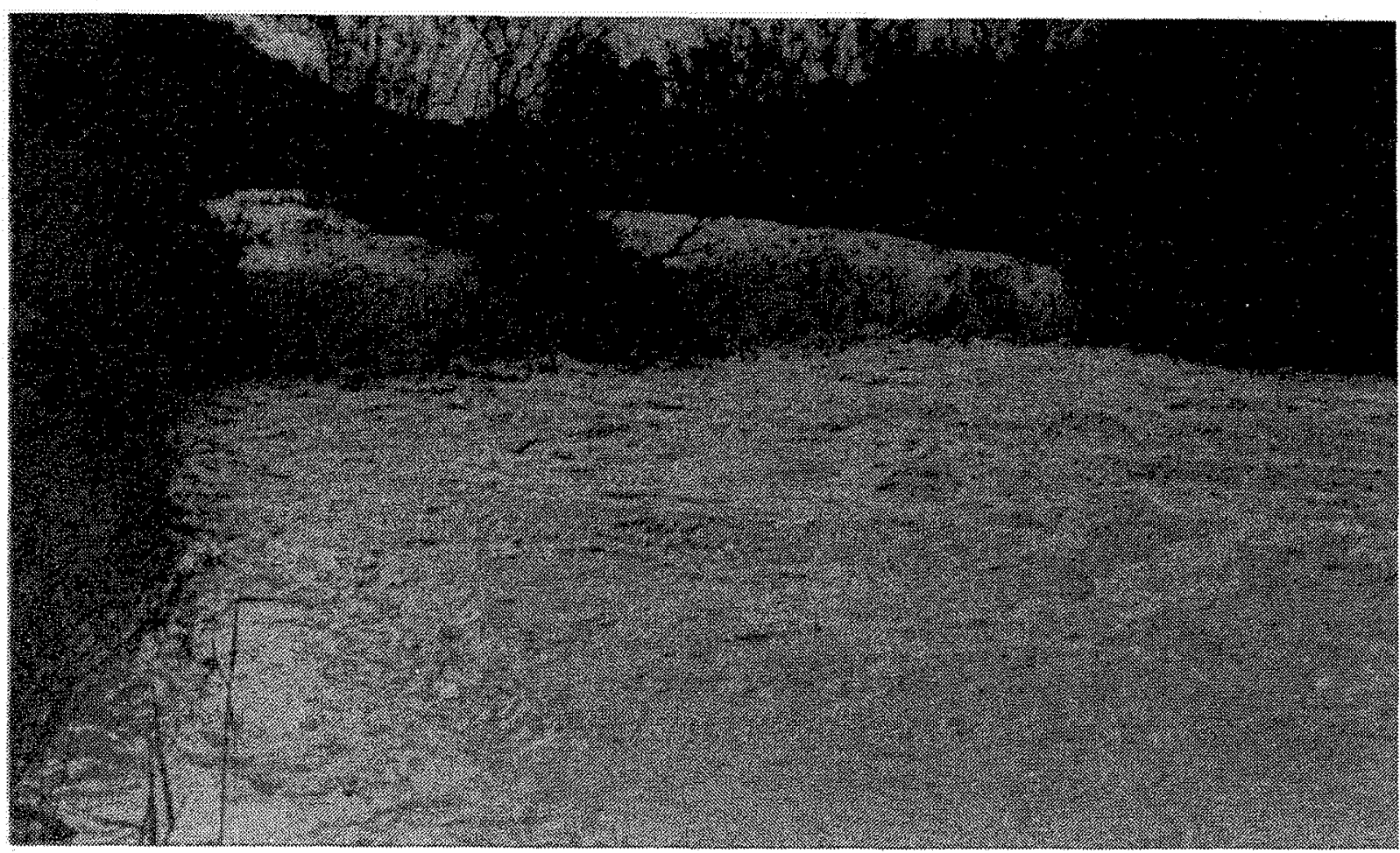

Figura 7. Salto producido en el lecho de Rambla Salada por avance de la erosión regresiva aguas arriba de la confluencia con el Barranco de la Zarza. 18 de Junio de 1997.

significativos, que básicamente contribuyen a crear estas discontinuidades. De hecho, los principales cambios observados en los tamaños del material de fondo son introducidos por los tributarios de Rambla Salada (Bco. de Fuente Higuera, Cañada Ramblizo y Bco. de la Zarza), que aportan sedimentos de muy diverso calibre.

Las obras de ingeniería civil realizadas en 1979 (el mencionado canal de regadio del Trasvase Tajo Segura) han introducido también importantes cambios morfosedimentarios. Una de estas obras auxiliares es el terraplén que cruza la Cañada Ramblizo, dotado de un pontón, cuya capacidad inicial de desagüe es inferior a la descarga dominante de la rambla. Con el incremento de la erosión por causa de una mayor inestabilidad del terreno removido (desmontes y taludes) y el efecto barrera impuesto por 'boulders' artificiales a la salida del tubo, la capacidad de éste puede disminuir sensiblemente a corto plazo y provocar una ruptura definitiva del anterior equilibrio dinámico del cauce. Otra importante modificación es el ensanchamiento de la sección del cauce principal de Rambla Salada, que acomoda los picos de avenida a la capacidad del vano del puente que la atraviesa. La interrupción de la llanura de inundación por el terraplén anexo a la estructura del puente, carente de vano de alivio, ha contribuido a cambios apreciables en la geometría de este cauce y en el comportamiento de la corriente.

Mayor impacto morfológico se observa en los tramos abarrancados afectados por las obras que acompañan el trazado del canal. La construcción, por ejemplo, de varias presas sobre el lecho del barranco de Los Guillermos ha alterado profundamente su régimen hidrológico, al tiempo que ha modificado su capacidad de transporte de sedimentos, acelerando los procesos de incisión aguas abajo (Conesa 
García et al., 1997). La profunda sobreexcavación remontante del antiguo lecho del barranco, lejos de ser un fenómeno aislado, afecta a distintos tributarios del Barranco de la Zarza sometidos al mismo tipo de alteración, poniendo de manifiesto la fragilidad del perfil de equilibrio de estos cursos.

\section{Conclusiones}

La cuenca de Rambla Salada, en el Sureste semiárido ibérico, aparece como un buen modelo para el estudio de las relaciones entre sus características biofísicas, usos del suelo y respuestas hidrológicas y geomorfológicas. Sin embargo, la información que hasta ahora suministra esta cuenca neógena es escasa debido a que el tiempo transcurrido desde el inicio de su instrumentalización (SAIH y campo experimental), operatividad y registro de datos es todavía corto. El objetivo de la red de sensores del dispositivo instrumental está orientado al pronóstico y evaluación hidrológica y erosiva mediante una utilización eficiente de la información.

Los datos suministrados por los sistemas de control y experiencias de campo, y la utilización de Modelos de Elevación Digital, integrados en un Sistema de Información Geográfica, son esenciales en aplicaciones geomorfológicas, hidrológicas y combinación de ambas, para la modelización de los flujos de la cuenca y cuantificación de las tasas de erosión. Los sistemas geomorfológicos e hidrológicos son variables en el tiempo y espacio, por lo que parece más adecuado que, en la cuenca de Rambla Salada, se utilicen modelos distribuídos antes que agregados para describir y evaluar su comportamiento hidrológico y producción de sedimentos.

Los valores de tasas de erosión, además del volumen de agua precipitado, energía de los aguaceros, topografia, resistencia mecánica de los materiales y usos del suelo, parecen depender, en gran medida, de la escala de observación y control. Por ello, se seguirá controlando la generación de escorrentías y pérdida de suelo a escala de parcela, y se afinará la evaluación de los flujos hídricos y la producción de sedimentos a escala de cuenca. La combinación de resoluciones espaciales en idéntica resolución temporal, podrá dar valores fiables del comportamiento hidrológico e importancia de los procesos de erosión en esta cuenca semiárida mediterránea.

\section{Agradecimientos}

La instrumentación y mantenimiento del Campo Experimental de Minglanillo es financiada por el convenio LUCDEME-RESEL de la Dirección General de Conservación de la Naturaleza (Ministerio de Medio Ambiente). El sistema SAIH es gestionado por la Confederación Hidrográfica del Segura. A ambos organismos expresamos nuestro agradecimiento.

\section{Bibliografia}

ALONSO SARRÍA, F., 1992. Estimación de la precipitación sobre cuencas hidrográficas durante episodios lluviosos de diversa duración. En Estudios de Geomorfología en España 
(F. López Bermúdez; Romero Díaz, M.A. y Conesa García, C., Eds.) Universidad de Murcia. pp. 151-160.

ALONSO SARRÍA,F. \& LÓPEZ BERMÚDEZ, F., 1994. Rainfall time and space variability during short storms in south-east Spain. Geoökodynamik, XV, 261-278

ALONSO SARRÍA, F., 1995: Análisis de episodios lluviosos y sus consecuencias bidrológicas sobre una cuenca mediterránea semiárida no aforada (Cuenca de la Rambla Salada, Murcia). Tésis Doctoral. Universidad de Murcia, $502 \mathrm{pp}$.

ALONSO SARRÍA, F., \& LÓPEZ BERMÚDEZ, F., 1998: Análisis topográfico e hidrogeomorfológico de la cuenca de la Rambla Salada (cuenca del Segura) a partir de un modelo Digital de Terreno. En Investigaciones recientes de la Geomorfología Española. (A. Gómez Ortiz, Salvador Franch, F., Schulte ,L. \& García Navarro, A., Eds.) Sociedad Española de Geomorfología. Universidad de Granada, pp. 697-700

CABEZAS, F, 1988. Modificación $N^{\circ} 1$ al proyecto Red Automática de Información Hidrológica de la Cuenca del Segura. Confederación Hidrográfica del Segura. Dirección General de Obras Hidráulicas. Ministerio de Obras Públicas y Urbanismo. Madrid.

CABEZAS, F., 1989. La problemática de las avenidas en la Cuenca del Segura. Criterios para la implantación del Sistema Automático de Información Hidrológica. En Avenidas fluviales e inundaciones en la cuenca mediterránea (A.Gil Olcina \& A.Morales, Eds.). Instituto Universitario de Geografia. Universidad de Alicante, Alicante, 491-500

C.H.S., 1992. SAIH. 60 Reunión Ejecutiva del Comité Internacional de Grandes Presas (CIGP). Granada, $10 \mathrm{pp}$.

CONESA GARCÍA, C. 1995. Torrential flow frequency and morphological adjustments of ephemeral channels in South-East Spain. In River Geomorpbology, ch. 9, (E.J. Hickin, Ed.), John Wiley \& Sons Ltd, Chichester, pp. 169-192.

CONESA GARCÍA, C., LÓPEZ BERMÚDEZ, F., ALONSO SARRIA, F. \& ALVAREZ ROGEL, Y., 1997. Hydraulic and Morphological Effects on the Derivation Tagus-Segura Works on the Ephemeral Channels in the Rambla Salada Basin (South-East Spain). Fourth International Conference on Geomorpbology, 28-VIII / 3-IX, 1997. Università di Bologna.

GARCÍA, S.G., 2000. Un Sistema de Simulación y Pronóstico Hidrológico Basado en GIS y DEM: Análisis de Sensibilidad a los Factores de Escala. Tesis Doctoral. Universidad Politécnica de Valencia. Escuela Técnica Superior de Ingenieros de Caminos, Canales y Puertos. Departamento de Ingeniería Hidráulica y Medio Ambiente. Valencia, 297 pp. más Anexos (inédita).

GRAF, W.L., 1988. Fluvial Processes in Dryland Rivers. Springer-Verlag. Berlin, 346 pp.

HARVEY, A.M, HITCHCOK, D. H. \& HUGUES, D. J., 1982. Event frequency and morphological adjusment of fluvial systems in Upland Britain. En: Adjustments of the Fluvial Systems (Rhodes, D.D. \& Williams, G.P., Eds), George Allen \& Unwin, London, 139-168.

LÓPEZ BERMÚdEZ, F., ALONSO SARRÍA, F., ROMERO DÍAZ, M.A.. CONESA GARCÍA, C., MARTÍNEZ FERNÁNDEZ, J. \& MARTÍNEZ FERNÁNDEZ, J., 1992. Caracterización y diseño del Campo Experimental de "Los Guillermos" (Murcia) para el estudio de los procesos de erosión y desertificación en litologías blandas. En Estudios de Geomorfología en España (López Bermúdez, F., Romero Díaz, M.A. \& Conesa García, C., Eds.) pp. 151-160.

LÓPEZ BERMÚDEZ, F., GARCÍA-RUIZ, J.M., ROMERO DÍAZ, M.A., RUIZ FLAÑO, P., MARTÍNEZ FERNÁNDEZ, J. \& LASANTA, T., 1993. Medidas de flujos de agua y sedimentos en parcelas experimentales. Sociedad Española de Geomorfología. Cuaderno Técnico $N^{\circ} 6$. Geoforma Ediciones. Logroño, $38 \mathrm{pp}$. 
LÓPEZ BERMÚDEZ, F., CONESA GARCÍA, C. \& ALONSO SARRÍA, F., 2000. Ramblas y barrancos mediterráneos: Medio Natural y respuesta humana. Finisterra. Lisboa, Portugal (En prensa).

PEDRERO GONZÁLEZ, A., 1993. Sistemas automáticos de información: el proyecto SAIH como sistema automático de información para la gestión de recursos hidraúlicos. En Conceptos y métodos para la planificación bidrológica. (J. Andreu, Ed.). Centro Internacional de Métodos Numéricos en Ingeniería (CIMNE). Barcelona, 83-96.

ROJO, L. \& SÁNCHEZ FUSTER, M.C., Coords., 1996. Red de estaciones experimentales de seguimiento y evaluación de la erosión y desertificación RESEL. Proyecto LUCDEME. Dirección General de Conservación de la Naturaleza. Ministerio de Medio Ambiente. Madrid, $121 \mathrm{pp}$.

ROMERO DÍAZ, M.A., ALONSO SARRÍA, F., FERNÁNDEZ GAMBÍN, I., LÓPEZ BERMÚDEZ, F. \& PÉREZ GARCÍA, J.A., 1998. Evolución de los usos del suelo y factores topográficos en la cuenca de la Rambla Salada (cuenca del Segura) a partir de un modelo Digital de Terreno. En Investigaciones recientes de la Geomorfología Española (A. Gómez Ortiz, F. Salvador Franch, L. Schulte \& A. García Navarro, Eds.). Universidad de Granada, pp. 751-756.

SCHMIDT, K.H. \& GINTZ, D., 1995. Results of Bedload Tracer Experiments in a Mountain River. En River Geomorphology, ch. 3. (Hickin, E.J., Ed.). John Wiley \& Sons Ltd, Chichester, pp. 37-54.

YAIR, A \& LAVEE, H. 1985. Runoff generation in arid and semi-arid zones. En Hydrological Forescastin (M.G. Anderson \& T.P.Burt, Eds.). John Wiley. Chichester: 183-220. 